\title{
DIMENSIONAMENTO DE PROFISSIONAIS DE ENFERMAGEM NOS SERVIÇOS HOSPITALARES COM ATENDIMENTO OBSTÉTRICO
}

\section{ARTIGO ORIGINAL}

LIMA, Thaís Bebiana de Almeida ${ }^{1}$

SOUZA, Karinne Ferreira de ${ }^{2}$

ALMEIDA, Eder Júlio Rocha de ${ }^{3}$

LIMA, Patrícia Oliveira ${ }^{4}$

ALMEIDA, Herlon Fernandes de ${ }^{5}$

LIMA, Thaís Bebiana de Almeida. Et al. Dimensionamento de profissionais de enfermagem nos serviços hospitalares com atendimento obstétrico. Revista Científica Multidisciplinar Núcleo do Conhecimento. Ano 05, Ed. 11, Vol. 06, pp. 0517. Novembro de 2020. ISSN: 2448-0959, Link de acesso: https://www.nucleodoconhecimento.com.br/saude/servicos-hospitalares

${ }^{1}$ Enfermeira, MBA em Gestão em Saúde e Administração Hospitalar, Especialista em Coaching em Enfermagem.

2 Orientadora. Mestre em administração, MBA em Auditoria e Gestão da Qualidade Aplicada aos Serviços de Saúde do Centro Universitário UNA.

${ }^{3}$ Doutorando em medicina; Mestre em tecnologia aplicada a saúde, MBA Gestão da Qualidade Aplicada aos Serviços de Saúde; especialista em urgência e emergência; Nefrologia; Segurança do paciente.

${ }^{4}$ Doutora em Odontologia, Mestre em ensino em saúde, enfermeira obstetra.

${ }^{5}$ Mestre em ensino em saúde, especialista em terapia intensiva de alta complexidade. 


\section{RESUMO}

As questões que envolvem a gestão em saúde estão cada vez mais evidenciadas no cotidiano dos profissionais da área. Como gestor de saúde, o enfermeiro necessita aplicar instrumentos gerenciais que possibilitem alcançar os objetivos propostos, para que a prática do cuidado seja efetiva e segura. Uma dessas ferramentas é o dimensionamento de pessoal na enfermagem. O objetivo desse estudo é identificar os parâmetros e instrumentos utilizados para realizar o dimensionamento das equipes de enfermagem em serviços de saúde hospitalares com atendimento obstétrico preconizado pelas recomendações, portarias e resoluções vigentes. Trata-se de uma pesquisa de revisão integrativa da literatura, de cunho qualitativo, utilizando resoluções e portarias do Ministério da Saúde e Conselho Federal de Enfermagem em vigor para direcionamento dos parâmetros de pessoal de enfermagem nesses serviços. Pode-se observar que para cada área de atuação de enfermagem no serviço de saúde foram identificadas portarias e resoluções que subsidiam gestores para a utilização de ferramentas de dimensionamento de pessoal de enfermagem, sendo a Resolução COFEN-543/2017 possível ser aplicada em todas as unidades da instituição onde há atuação da enfermagem. Nos cálculos devem ser considerados características dos serviços de saúde e garantia de requisitos mínimos estabelecidos pelo Ministério da Saúde. Conclui-se que os parâmetros e instrumentos apresentados, fornecem ferramentas para direcionar prestação de serviço seguro para os clientes das instituições, cabendo ao gestor a aplicação dos modelos de dimensionamento de pessoal de enfermagem rotineiramente correlacionando à carga de trabalho e indicadores de qualidade para a gestão dos serviços de saúde e garantia da sustentabilidade.

Palavras-Chave: Dimensionamento de pessoal, recursos humanos de enfermagem, gestão em saúde, administração hospitalar.

\section{INTRODUÇÃO}

As questões que envolvem a gestão em saúde estão cada vez mais evidenciadas no cotidiano dos profissionais da área, sendo objeto de vários estudos e propostas por 
parte de instituições formadoras ou de prestação de serviços (MAGALHÃES, 2009 apud MENEGUETI et al., 2013).

Uma das dimensões do trabalho do enfermeiro é gerenciar, e como gestor de saúde, o enfermeiro necessita aplicar instrumentos gerenciais que possibilitem alcançar os objetivos propostos, para que a prática do cuidado seja efetiva e segura.

Uma dessas ferramentas é o dimensionamento de pessoal na enfermagem, fundamental para realizar a previsão adequada de profissionais, quantitativa e qualitativamente, atendendo às necessidades da população assistida em consonância com a particularidade dos diversos cenários dos serviços de saúde, de forma a propiciar qualidade à assistência, visando à segurança dos usuários e dos trabalhadores (SANTOS et al., 2019).

De acordo com Santos, 2019, A equipe de enfermagem representa a maioria dos profissionais na área da saúde, tornando-se referência no que tange ao dimensionamento de mão de obra especializada, no âmbito hospitalar, respaldandose nas diretrizes e resoluções do Conselho Federal de Enfermagem (COFEN) de 1996, com a Resolução 189/1996, atualizada em 2004 (Resolução 293/2004) e em 2016 (Resolução 527/2016).

Se tratando do contexto hospitalar, há ainda outros parâmetros, encontrados nas resoluções do Ministério da Saúde que regulamentam as boas práticas dos serviços e estabelecem parâmetros mínimos de pessoal para determinados serviços.

Diante destas considerações, o presente estudo teve como objetivo identificar os parâmetros e instrumentos utilizados para o dimensionamento de pessoal de enfermagem em serviços de saúde hospitalares com atendimento obstétrico preconizado pelas recomendações, portarias e resoluções vigentes.

O estudo se justifica a partir da necessidade de existência de um único documento que contemple todas as referências necessárias para o dimensionamento de pessoal de enfermagem nos serviços de saúde hospitalares com atendimento obstétrico, auxiliando os gestores a estabelecer parâmetros adequados de pessoal de enfermagem, com embasamento nas recomendações, portarias e resoluções atuais 
para garantir a qualidade da assistência prestada, visando segurança dos pacientes e profissionais de enfermagem.

\section{METODOLOGIA}

Trata-se de uma pesquisa de revisão integrativa da literatura, de cunho qualitativo, sendo percorridas as seguintes etapas para elaboração: estabelecimento da hipótese ou questão da pesquisa; amostragem ou busca na literatura; categorização dos estudos; avaliação dos estudos incluídos na revisão; interpretação dos resultados e síntese do conhecimento ou apresentação da revisão (MENDES; SILVEIRA; GALVÃO, 2008).

Na primeira fase foi definido o tema e elaborado o objetivo geral, a questão norteadora de pesquisa configura na seguinte pergunta: Quais os parâmetros e instrumentos utilizados para o dimensionamento de pessoal de enfermagem em serviços de saúde hospitalares preconizado pelas recomendações, portarias e resoluções vigentes?

Para embasamento teórico foi realizado uma busca na plataforma eletrônica Biblioteca Virtual em Saúde (BVS), que permitiu o acesso à base de dados da Literatura LatinoAmericana em Ciências da Saúde (LILACS), Base de dados em Enfermagem (BDENF) e Literatura Internacional em Ciências da Saúde (MEDLINE) através dos seguintes descritores: Dimensionamento de pessoal; Recursos humanos de enfermagem; Gestão em Saúde; Administração Hospitalar.

Como critério de inclusão utilizou-se o recorte temporal de 2013 a 2019, artigos completos nos idiomas portuguesa e inglesa que contemplarem o tema ora proposto. Os critérios de exclusão estabelecidos foram estudos anteriores a 2013, não disponíveis na integra e gratuitos.

Avaliou-se inicialmente o título e o resumo dos 30 artigos científicos encontrados, pois contemplavam o tema a ser discutido e propiciaram uma maior capacidade de investigação. Após leitura e análise minuciosa, 3 artigos científicos foram selecionados. 
Optou-se pelo uso do quadro sinóptico para compilar as seguintes informações referente os artigos selecionados: título do artigo, autoria, periódico (vol., ํo, pg., ano, revista publicada), resultados, síntese das conclusões/recomendações.

Além dos artigos, foram utilizadas resoluções e portarias do Ministério da Saúde e Conselho Federal de Enfermagem em vigor para direcionamento dos parâmetros de pessoal de enfermagem.

\section{REFERENCIAL TEÓRICO}

A Resolução COFEN-543/2017 estabelece os parâmetros mínimos para dimensionar - quantitativo de profissionais das diferentes categorias de enfermagem para hospitais, clínicas e laboratórios que são prestados serviços de enfermagem.

Os parâmetros representados nas normas técnicas mínimas, constituindo-se em referências para orientar os gestores, gerentes e enfermeiros dos serviços de saúde, no planejamento do quantitativo de profissionais necessários para execução das ações de enfermagem (COFEN, 2017).

O dimensionamento das equipes de enfermagem deve basear-se em características relativas ao serviço de saúde, requisitos mínimos estabelecidos pelo Ministério da Saúde, ao serviço de enfermagem e ao paciente (COFEN, 2017).

A Resolução RDC no 36/2008 dispõe sobre Regulamento Técnico para Funcionamento dos Serviços de Atenção Obstétrica e Neonatal, estabelecendo que o Serviço de Atenção Obstétrica e Neonatal deve ter equipe dimensionada, quantitativa e qualitativamente, atendendo as normatizações vigentes, e de acordo com a proposta assistencial e perfil de demanda (BRASIL, 2008). 


\subsection{UNIDADES DE INTERNAÇÃO (ALOJAMENTO CONJUNTO) E UNIDADE DE CUIDADOS INTERMEDIÁRIOS NEONATAL CONVENCIONAL (UCINCO)}

A UCIN compõem uma linha de cuidado progressivo, que visam a equilibrar a necessidade do recém-nascido com a capacidade clínica que está instalada, e recebe a classificação em diversos níveis: decrescente de complexidade como UTI neonatal, Podendo ser também classificado como de cuidados intermediários neonatais (UCIN) e de Alojamento Conjunto(ALCON). foi criada sob a égide da Portaria no 1091/GM de 25 de agosto de 1999, revogada pela Portaria № 930, de 10 de maio de 2012.

Que estabelece as diretrizes e objetivos para a organização da atenção integral e humanizada ao recém-nascido grave ou potencialmente grave e os critérios de classificação e habilitação de leitos de Unidade Neonatal no âmbito do Sistema Único de Saúde (SUS). Em seu Artigo 17 dispões sobre a formação da equipe de enfermagem sendo: 1 (um) enfermeiro coordenador preferencialmente com habilitação em neonatologia ou no mínimo 2 anos de experiência profissional comprovada, com jornada horizontal diária mínima de 4 (quatro) horas, 1 (um) enfermeiro assistencial, para cada 15 (quinze) leitos ou fração, em cada turno e 1 (um) técnico de enfermagem para cada 5 (cinco) leitos, em cada turno. (BRASIL, 2012).

A Portaria № 2068 de 21 de outubro de 2016, institui diretrizes para acontecer a prática da atenção integrativa e humanizada à mulher e ao recém-nascido no Alojamento Conjunto. Dispõe em seu Art. 5o a respeito dos recursos humanos mínimos necessários.

Para Enfermagem recomenda-se: profissional de nível superior para função de coordenação, preferencialmente com habilitação em neonatologia/obstetrícia ou 2 (dois) anos de experiência profissional comprovada na área, com jornada horizontal diária mínima de quatro horas, profissional de nível superior para assistência, preferencialmente com habilitação em neonatologia/obstetrícia ou 2 (dois) anos de experiência profissional comprovada na área para cada 20 binômios mãe-RN ou fração em cada turno; e profissional de nível técnico para cada oito binômios mãe-RN ou fração em cada turno (BRASIL, 2016). 
O Sistema de classificação de pacientes (SCP) é uma ferramenta que predispõe a dependência da equipe de enfermagem, e assim, podendo mensurar o quantitativo de horas de assistência prestada de maneira direta e indireta, podendo melhorar a qualidade das intervenções biopsicosocioespirituais dos clientes. Através do SCP é possível classificar o paciente em "cuidados mínimos", "cuidados intermediários", "cuidados de alta dependência", "cuidados semi-intensivo" e "cuidados intensivos" (COFEN, 2017).

A referida resolução prevê o percentual a ser distribuído de profissionais de enfermagem, conforme o SCP (Sendo função privativa do enfermeiro o registro diário do score obtido na classificação dos pacientes) e as proporções mínimas, bem como a proporção profissional/paciente nos diferentes turnos de trabalho:

Para cuidado mínimo: 33\% são enfermeiros (mínimo de seis) e os demais auxiliares e/ou técnicos de enfermagem, 1 profissional de enfermagem para 6 pacientes; Para cuidado intermediário: 33\% são enfermeiros (mínimo de seis) e os demais auxiliares e/ou técnicos de enfermagem, 1 profissional de enfermagem para 4 pacientes; Para cuidado de alta dependência: $36 \%$ são enfermeiros e os demais técnicos e/ou auxiliares de enfermagem, 1 profissional de enfermagem para 2,4; Para cuidado semi-intensivo: $42 \%$ são enfermeiros e os demais técnicos de enfermagem, 1 profissional de enfermagem para 2,4; Para cuidado intensivo: $52 \%$ são enfermeiros e os demais técnicos de enfermagem, 1 profissional de enfermagem para 1,33 (COFEN, 2017).

Para cálculo de pessoal da Resolução COFEN-543/2017 em unidades de internação e Unidades de Cuidados Intermediários Neonatal, há a fórmula de cálculo com base na relação de proporção profissional/pacientes, devendo classificar os pacientes de alojamento conjunto (binômio mãe/filho), berçário e unidade de internação em pediatria (todo recém-nascido e criança menor de 6 anos), no mínimo como cuidado intermediário: 
1. Método de cálculo de mão de obra para binômio.

$$
\mathrm{QP}_{(\mathrm{UI})}=\left\{\left[\left(\frac{\mathrm{PCM}}{6}\right)+\left(\frac{\mathrm{PCI}}{4}\right)+\left(\frac{\mathrm{PCAD}}{2,4}\right)+\left(\frac{\mathrm{PCSI}}{2,4}\right)+\left(\frac{\mathrm{PCIt}}{1,33}\right)\right] \times\left(\frac{\mathrm{PF} \times \mathrm{DS}}{\mathrm{CHS}}\right) \times(1+\mathrm{IST})\right\}
$$

Onde:

PF = periodo de funcionamento da unidade ( 24 horas);

DS = dias da semana (7 dias);

$\mathrm{CHS}=$ carga horária semanal.

$(1+I S T)=$ Fator de ajuste do índice de segurança técnica.

Fonte: COFEN,2017.

\subsection{CENTRO CIRÚRGICO / OBSTÉTRICO}

Para centro cirúrgico / obstétrico é possível utilizar como referência para a estimativa de equipe de enfermagem os princípios da RDC no 36/2008 já citado neste artigo e a Resolução COFEN-543/2017.

O referencial mínimo para o quadro dos profissionais de enfermagem em Centro Cirúrgico, Leva em consideração a ASA cirúrgica, o porte cirúrgico (porte 1 a porte 4 conforme a duração do procedimento), o tempo de limpeza das salas $(0,5$ horas para eletivas e 0,6 para urgência e emergência) e o tempo de espera das cirurgias $(0,2$ horas por cirurgia).

Como proporção profissional/categoria, nas 24 horas: 1 (um) enfermeiro para cada três salas cirúrgicas (eletivas); Enfermeiro exclusivo nas salas de cirurgias eletivas e de urgência/emergência de acordo com o grau de complexidade e porte cirúrgico; 1 (um) profissional técnico/auxiliar de enfermagem para cada sala como circulante (de acordo com o porte cirúrgico); e 1 (um) profissional técnico/auxiliar de enfermagem para a instrumentação (de acordo com o porte cirúrgico) (COFEN, 2017). 
2. Cálculo de mão de obra em centro cirúrgico.

Cálculo do THE para procedimentos (Intervenções/atividade)

$$
\mathrm{THE}=(\mathrm{P} 1 \times \mathrm{H} 1)+(\mathrm{P} 2 \times \mathrm{H} 2)+(\mathrm{P} 3 \times \mathrm{H} 3)+(\mathrm{P} 4 \times \mathrm{H} 4)
$$

Onde:

$\mathrm{THE}=$ total de horas de enfermagem para realização da programação cirúrgica;

$\mathrm{P}_{(1,2,3,4)}=$ número médio de cirurgias segundo o porte cirúrgico.

$\mathrm{H}_{(1,2,3,4)}=$ tempo médio por cirurgia segundo porte, tempo de limpeza e de espera.

Fonte: COFEN,2017.

\subsection{CENTRAL DE MATERIAIS E ESTERILIZAÇÃO (CME)}

A resolução RDC 15 de março de 2012, que regulamenta o funcionamento da Central de Materiais Esterilizados não dispõe acerca do quantitativo de pessoal necessário para exercer as atividades do setor, no entanto dispõe a respeito da necessidade de um enfermeiro exclusivo e que os colaboradores sejam capacitados para exercer a função.

A RESOLUÇÃO COFEN-0543/2017 estabeleceu em 2017 que a jornada laboral da enfermagem para a unidade Central de Materiais e Esterilização (CME) devendo levar em consideração o quantitativo produzidos representados por unidades, multiplicada pelo tempo das atividades realizadas, nas diferentes áreas: 
3. Descrição de atividades por sítio funcional

\begin{tabular}{|c|c|c|c|}
\hline \multirow[t]{2}{*}{$\overline{\text { AREA }}$} & \multirow[t]{2}{*}{ DE SCRIÇÁAO DE ATIMDADE S } & \multicolumn{2}{|c|}{$\begin{array}{l}\text { TEMPO } \\
\text { PADRÃO }\end{array}$} \\
\hline & & Minuto & Hora \\
\hline \multirow[t]{2}{*}{$\begin{array}{l}\text { Suja ou contam inada } \\
\text { (expurgo) }\end{array}$} & $\begin{array}{l}\text { Recepção e recolhimento dos materiais } \\
\text { contaminados* }\end{array}$ & 2 & 0,033 \\
\hline & Limpeza dos materiais * & 2 & 0,033 \\
\hline \multirow{3}{*}{$\begin{array}{lr}\text { Controle } & \text { de } \\
\text { materiais } & \text { em } \\
\text { consignação } & \end{array}$} & $\begin{array}{l}\text { Recepção dos materia is em } \\
\text { consignação * }\end{array}$ & 6 & 0,1 \\
\hline & $\begin{array}{l}\text { Conferência dos Materiais Consignados } \\
\text { após cirurgia * }\end{array}$ & 9 & 0,15 \\
\hline & $\begin{array}{l}\text { Devolução dos materiais em } \\
\text { consignação * }\end{array}$ & 3 & 0,05 \\
\hline \multirow{4}{*}{ Preparo de materiais } & $\begin{array}{l}\text { Secagem e distribuição dos materiais } \\
\text { após limpeza * }\end{array}$ & 3 & 0,05 \\
\hline & $\begin{array}{l}\text { Inspeção, teste, separação e secagem } \\
\text { dos materiais * }\end{array}$ & 3 & 0,05 \\
\hline & Montagem e embalagem dos materiais * & 3 & 0,05 \\
\hline & $\begin{array}{l}\text { Montagem dos materiais de assistência } \\
\text { ventilatória : }\end{array}$ & 2 & 0,033 \\
\hline \multirow{2}{*}{$\begin{array}{l}\text { Esterilização } \\
\text { de materiais }\end{array}$} & Montagem da carga de esterilização ** & 8 & 0,133 \\
\hline & $\begin{array}{l}\text { Retira da da carga estéril e verificação da } \\
\text { esterilização ** }\end{array}$ & 3 & 0,05 \\
\hline \multirow{4}{*}{$\begin{array}{l}\text { Armazenamento e } \\
\text { distribuiçäo de } \\
\text { materiais }\end{array}$} & Guarda dos Materiais ** & 4 & 0,066 \\
\hline & $\begin{array}{l}\text { Montagem dos carros de transporte das } \\
\text { unidades } * * *\end{array}$ & 5 & 0,083 \\
\hline & $\begin{array}{l}\text { Organização e controle do ambiente e } \\
\text { materiais estéreis * }\end{array}$ & 1 & 0,016 \\
\hline & $\begin{array}{l}\text { Distribuição dos materiais e roupas } \\
\text { estéreis * }\end{array}$ & 2 & 0,033 \\
\hline
\end{tabular}

Fonte: COFEN,2017.

\subsection{CENTRO DE TERAPIA INTENSIVA (CTI)}

A resolução RDC 07/2010 regulamenta os requisitos mínimos para funcionamento de Unidades de Terapia Intensiva, e assim dispõe sobre a composição da equipe de enfermagem, sendo necessário 1 (um) um enfermeiro coordenador da equipe de enfermagem, enfermeiros assistenciais no mínimo 01 (um) para cada 08 (oito) leitos por jornada. 
Técnicos de enfermagem no mínimo 01 (um) para cada 02 (dois) leitos em cada turno, além de 1 (um) técnico de enfermagem por UTI para serviços de apoio assistencial em cada turno (BRASIL, 2010).

Tal regulamento foi alterado em 2012 com a publicação da RDC 26/2012, alterando o quantitativo de enfermagem para: Enfermeiros no mínimo 01 (um) para cada 10 (dez) leitos, em cada turno, Técnicos de enfermagem: no mínimo 01 (um) para cada 02 (dois) leitos em cada turno (BRASIL, 2012).

A Portaria № 930, de 10 de maio de 2012,que define as diretrizes e objetivos para a organização da atenção integral e humanizada ao recém-nascido grave ou potencialmente grave e os critérios de classificação e habilitação de leitos de Unidade Neonatal no âmbito do Sistema Único de Saúde (SUS). Para unidade de terapia intensiva neonatal (UTIN), há duas habilitações sendo UTIN tipo II ou UTIN tipo III. Para habilitação de UTIN tipo II a equipe de enfermagem deve ser formada minimamente por 1 (um) enfermeiro coordenador com jornada horizontal diária de 8 horas com habilitação em neonatologia ou no mínimo 2 (dois) anos de experiência profissional comprovada em terapia intensiva pediátrica ou neonatal; 1 (um) enfermeiro assistencial para cada 10 (dez) leitos ou fração, em cada turno; e técnicos de enfermagem, no mínimo, 1 (um) para cada 2 (dois) leitos em cada turno. Para habilitação de UTIN do tipo III, o serviço hospitalar deverá contar com toda a estrutura prevista no tipo II e enfermeiro coordenador com título de especialização em terapia intensiva/terapia intensiva neonatal ou no mínimo 5 (cinco) anos de experiência profissional comprovada de atuação na área e 1 (um) enfermeiro plantonista assistencial por turno, exclusivo da unidade, para cada 5 (cinco) leitos ou fração (BRASIL, 2012).

Para cálculos relacionados ao quantitativo de pessoal de enfermagem, é possível utilizar a mesma lógica descrita no item Unidade de Internação, realizando o cálculo recomendado na Resolução COFEN 0543/2017 após determinar a classificação dos pacientes utilizando o SCP.

\section{RESULTADOS E ANÁLISE DOS DADOS}

Em relação do dimensionamento de pessoal de enfermagem, pode-se observar que em Unidades de Internação (Alojamento Conjunto) e Unidade de Cuidados Intermediários Neonatal Convencional, é possível dimensionar pessoal de 
enfermagem utilizando como embasamento duas portarias do Ministério da Saúde (№ 930/2012 e № 2068/2016).

Para centro cirúrgico / obstétrico é possível utilizar como referência os princípios da Resolução RDC no 36/2008 e para Central de Materiais e Esterilização a Resolução RDC nº15/2012 do Ministério da Saúde.

Nas unidades de terapia intensiva pode-se dimensionar o pessoal de enfermagem utilizando os preceitos da Resolução RDC 07/2010, e quando se tratar de UTI Neonatal, a portaria № 930/2012 do Ministério da Saúde.

A Resolução COFEN-543/2017 é possível ser aplicada em todas as unidades da instituição onde há atuação da enfermagem. Estabelece diretrizes para definir a proporção profissional/paciente nos diferentes turnos de trabalho para as unidades assistenciais com utilização do SCP, considera a classificação da cirurgia e tempos para assistência, limpeza e espera para o dimensionamento em centro cirúrgico e obstétrico, e fornece fundamentação para cálculo de pessoal baseado na produção e tempo para o processamento dos materiais na Central de Materiais e Esterilização (CME).

Em todos os cálculos para dimensionamento de pessoal devem ser considerados as características dos serviços de saúde com a utilização da taxa de ocupação da instituição e garantia de requisitos mínimos estabelecidos pelo Ministério da Saúde.

\section{CONSIDERAÇÕES FINAIS}

Conclui-se que os parâmetros e instrumentos utilizados para o dimensionamento de pessoal de enfermagem em serviços de saúde hospitalares com atendimento obstétrico preconizado pelas recomendações, portarias e resoluções vigentes, fornecem ferramentas para direcionar prestação de serviço seguro para os clientes das instituições, sendo necessário ressaltar que para todas as áreas apresentadas, as características do serviço de saúde devem ser analisadas. Além da segurança, deve-se considerar o efetivo controle de custos, cabendo ao gestor a aplicação dos 
modelos de dimensionamento de pessoal de enfermagem rotineiramente correlacionando à carga de trabalho e indicadores de qualidade para a gestão dos serviços de saúde e garantia da sustentabilidade.

\section{REFERÊNCIAS}

BRASIL. Ministério da Saúde. Portaria no 2068, de 21 de outubro de 2016. Institui diretrizes para a organização da atenção integral e humanizada à mulher e ao recémnascido no Alojamento Conjunto.

BRASIL. Ministério da Saúde. Portaria № 930, de 10 de maio de 2012. Define as diretrizes e objetivos para a organização da atenção integral e humanizada ao recémnascido grave ou potencialmente grave e os critérios de classificação e habilitação de leitos de Unidade Neonatal noâmbito do Sistema Único de Saúde (SUS).

BRASIL. Ministério da Saúde. RESOLUÇÃO - RDC № 26, DE 11 DE MAIO DE 2012. Altera a Resolução RDC №. 07, de 24 de fevereiro de 2010, que dispõe sobre os requisitos mínimos para funcionamento de Unidades de Terapia Intensiva e dá outras providências.

BRASIL. Ministério da Saúde. RESOLUÇÃO - RDC № $\mathbf{3 6}$ de 03 de junho de 2008. Dispõe sobre Regulamento Técnico para Funcionamento dos Serviços de Atenção Obstétrica e Neonatal

BRASIL. Ministério da Saúde. Resolução da diretoria colegiada RDC oㅜ 15, de 15 de março de 2012. Dispõe sobre requisitos de boas práticas para o processamento de produtos para saúde e dá outras providências.

BRASIL. Ministério da Saúde. Resolução № 7, DE 24 DE FEVEREIRO DE 2010. Dispõe sobre os requisitos mínimos para funcionamento de Unidades de Terapia Intensiva e dá outras providências.

COFEN. Conselho Federal de Enfermagem. RESOLUÇÃO COFEN-0527/2016. Atualiza e Estabelece Parâmetros para o Dimensionamento do Quadro de 
Profissionais de Enfermagem nos serviços/locais em que são realizadas atividades de enfermagem.

COFEN. Conselho Federal de Enfermagem. RESOLUÇÃO COFEN-0543/2017. Atualiza e Estabelece Parâmetros para o Dimensionamento do Quadro de Profissionais de Enfermagem nos serviços/locais em que são realizadas atividades de enfermagem.

COFEN. Conselho Federal de Enfermagem. RESOLUÇÃO COFEN-293/2004. Fixa e Estabelece Parâmetros para o Dimensionamento do Quadro de Profissionais de Enfermagem nas Unidades Assistenciais das Instituições de Saúde e Assemelhados.

MENDES, Karina Dal Sasso; SILVEIRA, Renata Cristina de Campos Pereira; GALVAO, Cristina Maria. Revisão integrativa: método de pesquisa para a incorporação de evidências na saúde e na enfermagem. Texto contexto - enferm., Florianópolis , v. 17, n. 4, p. 758-764, Dec. 2008 . Disponível em: $<$ http://www.scielo.br/scielo.php?script=sci arttext\&pid=S0104-

07072008000400018\&lng=en\&nrm=iso $>$. Acesso em: 23 de julho de 2019.

MENEGUETI, Mayra Gonçalves; NICOLUSSI, Adriana Cristina; SCARPARO, Ariane Fazzolo; CAMPOS, Luciana de Freitas; CHAVES, Lucieli Dias Pedreschi; LAUS, Ana Maria. Dimensionamento de pessoal de enfermagem nos serviços hospitalares: revisão integrativa da literatura. Revista Eletrônica de Enfermagem, vol 15 (2), 2013. Disponível em: < https://www.fen.ufg.br/revista/v15/n2/pdf/v15n2a30.pdf >. Acesso em: 23 de julho de 2019.

SANTOS, Lucas Cardoso dos; ANDRADE, Juliane; SPIRI, Wilza Carla. Dimensionamento de profissionais de enfermagem: implicações para o processo de trabalho na estratégia saúde da família. Esc Anna Nery, volume 23 (3), 2019. Disponível em: < http://www.scielo.br/pdf/ean/v23n3/pt_1414-8145-ean-2303-e20180348.pdf>. Acesso em: 23 de julho de 2019.

Enviado: Novembro, 2020. 
Aprovado: Novembro, 2020. 